\title{
Minocycline Attenuates Kidney Injury in a Rat Model of Streptozotocin-Induced Diabetic Nephropathy
}

\author{
Hong-ping YUAN ${ }^{1}$, Dong-xu ZHAO ${ }^{2,{ }^{*}}$ and Bo-yin $\mathrm{ZHANG}^{2}$ \\ ${ }^{1}$ Department of nephrology, the Fourth Hospital of Jilin University, 2643 Dongfeng \\ Road, Changchun 130011, China. \\ ${ }^{2}$ China-Japan Union Hospital of Jilin University, 126 Xian-Tai Street, Chang Chun \\ 130033,China.
}

${ }^{*}$ Corresponding author

Keywords: Minocycline, Kidney injury, Rat model, Diabetic nephropathy.

\begin{abstract}
The effects of minocycline on the development of diabetic nephropathy (DN) in streptozotocin (STZ) induced diabetic rats were evaluated in this study. The diabetes rats with diabetic nephropathy (DN) were induced by STZ $(55 \mathrm{mg} / \mathrm{kg})$ injection. The experiment included 5 groups 1) normal, 2) normal plus minocucline for 16 weeks, 3) DN plus vehicle, 4) DN plus minocucline 16 weeks and 5) DN plus minocucline for 8 weeks. The pathological changes were analyzed by H.E. staining and the apoptotic cells were stained by TUNEL staining. The mRNA expression of caspase-3, bax and bcl-2 in the kidney tissues was detected by quantitative RT-PCR. The biochemical parameters of blood and urine were determined by biochemical analyzer. Treatment with minocycline reduced the urine volume, 24-hour urine protein, serum creatinine, blood urea nitrogen but not blood ALT in the DN rats. Furthermore, treatment with minocycline improved the pathological score of STZ-injured kidney and reduced the numbers of apoptotic cells in the kidney of DN rats. Moreover, minocycline mitigated the expression of caspase- 3 and bax mRNA, but increased Bcl-2 expression in the kidney of DN rats. These data indicated that minocycline improved the STZ-induced kidney damages, at least partially by protection form long-term hyperglycemia-induced kidney cell apoptosis.
\end{abstract}

\section{Introduction}

Diabetes mellitus is a complex disease that causes altered glucose homeostasis[1] and diabetic nephropathy (DN) is the major microvascular complication of diabetes which is prevalent among diabetic patients[2]. It is estimated that DN progresses to end-stage renal disease in up to $30 \%$ of patients with diabetes. DN is currently the leading cause of end-stage renal disease in most developed societies and is increasingly prevalent in developing countries. The clinical characteristics of DN are progressive urinary albumin excretion and gradual decline of renal function, which results from the multiple pathophysiologic dysfunctions of diabetes. The appearance of microalbuminuria is regarded as the hallmark of DN onset at its early stage, and the continuous existence of albuminuria plays a crucial role in the damage of both renal structure and function. Although the treatment and understanding of DN are progressing, in clinical practice, the strict control of glucose and blood pressure, and the blockade of the renin-angiotensin system can only slow the progression of DN, however, no available therapy can cure DN. 
The increased urinary albumin excretion is due to the dysfunction of the glomerular barrier, which is considered to be a key target for the prevention and treatment of DN. Among the elementary ingredients of glomeruli structure, the podocyte and its foot process play the pivotal role in maintenance of permselective function of the glomerular barrier[3].

Minocycline is a semi-synthetic derivative of tetracycline and has been used as antibiotic for decades. Both in vivo and in vitro data indicate that podocyte apoptosis is a hallmark of albuminuria[4,5]. Clinical biopsy studies in humans have also provided evidence that podocytes are functionally and structurally injured during the progression of DN [3]. The loss of glomerular podocytes and decreased podocyte density are early pathological manifestations and predict the onset of $\mathrm{DN}[3,6]$. Podocyte loss precedes the development of comprehensive renal dysfunction and albuminuria in diabetic patients and animal models[7]. Podocyte loss resulting from apoptosis has played an important role in the onset of albuminuria and the pathogenesis of $\mathrm{DN}[8]$. Recent studies demonstrate that preventing podocyte apoptosis may ameliorate renal injury and decrease proteinuria in DN $[9,10]$. Recent studies have shown that anti-inflammatory and immunomodulatory actions of minocycline are not related to its antimicrobial property[11]. Studies have also suggested a neuroprotective effect of minocycline in experimental models of ischemic stroke[12]. Huntington's disease[13]. Parkinson's disease[14] traumatic brain injury[15] amyotropic lateral sclerosis[16] and multiple sclerosis[17].The documented direct neuronal protection by minocycline is associated with the preservation of mitochondrial integrity and cytochrome $\mathrm{c}$, and subsequent the suppression of caspase-dependent as well as -independent cell death[16,18,19]. Wang et al. reported that minocycline protects kidney epithelial cells against injury and death through the protection of the mitochondrion[20]. Furthermore, they suggest that Bcl-2 is a key molecular determinant of the cytoprotective effects of minocycline and down-regulation of $\mathrm{Bcl}-2$ by antisense oligonucleotides abolishes the cytoprotective effects of minocycline. Moreover, the anti-inflammatory and immunomodulatory actions of minocycline may be attributed to the inhibition of free radical and cytokine production, interference with protein synthesis, and modulation of matrix metalloproteinase activity[21,22]. Interestingly, previous studies have also shown that tetracyclines possess potent antiapoptotic properties by inhibition of caspase 1 and 3 expression and direct blockade of cytochrome $\mathrm{c}$ release from the mitochondria[13,16]. Thus, minocycline, a semisynthetic derivative of tetracycline, offers a unique and potentially approach to the prevention and treatment of DN by its anti-apoptotic effects as investigated in this paper.

\section{Materials and Methods}

\section{Animals}

Male Wistar rats at 6-8 weeks of age and weighing 200-250 g, were from the Experimental Animal Center, Jilin University, China. Animals were housed in specific pathogen free facility with free access of normal chow and water ad libitum at $22^{\circ} \mathrm{C}$ and a cycle of 12 hours of light and dark. The experimental protocol was established, according to the guidelines for the use and care of laboratory animals at Jilin University, and was approved by the Animal Research and Care Committee of Jilin University. 


\section{Induction of Diabetes}

Animals were fasted overnight and injected intraperitoneally with streptozotocin (STZ, 55 $\mathrm{mg} / \mathrm{kg}$ of body weight, Sigma, St. Louis, USA). Control rats received vehicle alone. Two days after STZ injection, the levels of fasting blood glucose (FBG) in individual rats were measured. Individual rats with a FBG $>16.7 \mathrm{mmol} / \mathrm{L}$ were considered diabetic. The diabetes rats were monitored for their body weights, FBG, urinary albumin and urinary proteins for 16 weeks. Individual diabetic rats with urinary albumin $>200 \mu \mathrm{g} / \mathrm{min}$ or urinary proteins $>0.5 \mathrm{~g} / 24 \mathrm{~h}$ were diagnosed as diabetic nephropathy (DN).

All control and DN rats were randomly treated with saline or treated orally with minocycline $(20 \mathrm{mg} / \mathrm{Kg}$ ) by gavage daily for 16 weeks as the healthy controls (HC), healthy minocycline (HM), DN and diabetic minocycline (M4), respectively. Another group of DN rats were treated with the same dose of minocycline from the 9th to 16th weeks (M2)

\section{Sample Collection and Analysis}

For collection of 24-hour urine, animals were housed in metabolic cages for 24 hours with free access of water at the 4th, 8th, 12th and 16th week post the onset hyperglycemia. The collected urine samples were measured for their volumes and urinary proteins by a dipstick dye immunoassay (Shenhua biotech, Jilin, China). Subsequently, the urinary samples were centrifuged at 2,000 $\mathrm{g}$ for $10 \mathrm{~min}$ at room temperature and the supernatants were stored at $-70^{\circ} \mathrm{C}$ until use. At the end of this experiment, the rats were anesthetized with thiopental sodium (60 mg/kg. i.p.), and their blood samples were collected in serum-separating tubes (SSTs) and centrifuged at $2,000 \mathrm{~g}$ for $20 \mathrm{~min}$ at $4^{\circ} \mathrm{C}$. The serum samples from individual rats were stored at $-70^{\circ} \mathrm{C}$ until use. The concentrations of serum BUN, creatinine (Scr) and alanine aminotransferase (ALT) were detected with a HITACHI automatic biochemical analyzer using commercial kits (HITACHI, Japan).

\section{Histology and Terminal Deoxynucleotidyl Transferase-Mediated Dutp Nick Endlabeling (TUNEL) Assay}

Their kidney tissues were dissected, fixed and embedded in paraffin. The kidney tissue sections $(3 \mu \mathrm{m})$ were stained with hematoxyline/eosin and PAS, followed examining under a light microscope. The glomerular areas in five sections of individual kidney tissues were measured. The apoptotic cells in kidney sections were determined by TUNEL assays using an in situ apoptosis detection kit (Roche, Germany), according to manufacturer's protocol. The percentages of apoptotic cells were calculated.

\section{Quantitative Real Time Reverse Transcriptase PCR}

Total RNA of tissues was extracted from fresh kidney tissues from individual rats using Trizol reagent (Invitrogen), according to the manufacturer's instruction. After quality and quantification, RNA (5 $\mu$ g per sample) was reversely transcribed into cDNA using random hexamer primers and Rnase H-reverse transcriptase (Tiangen biochen, Beijing, China). The relative levels of Bcl-2, Bax, caspase-3 mRNA transcripts to control GAPDH were determined by quantitative PCR using the SYBR green System and specific primers. The sequences of primers were F: 5-CCGGGAGAACAGGGTATGAT-3 and R: 5-CAGGTATGCACCCAGAGTGA-3 for Bcl-2 (88bp); F: 5-TCAGCCCATCTTCTTCCAGATGGT-3, R:5-CCACCAGCTCTGAACAGATCATGA-3for Bax (135bp); F: 
5-GGTATTGAGACAGACAGTGG-3, and R: 5-CATGGGATCTGTTTCTT TGC-3 for Caspase-3 (281 bp); F: 5- AAGGTCATCCCAGAGCTGAACGG -3', and R: 5ACAACCTGGTCCTCAGTGTAGCC-3 for GAPDH (191bp). The PCR amplification was performed in triplicate at $50^{\circ} \mathrm{C}$ for $2 \mathrm{~min}, 95^{\circ} \mathrm{C}$ for $10 \mathrm{~min}$, and subjected to 40 cycles of $95^{\circ} \mathrm{C}$ for $30 \mathrm{~s}$ and $60^{\circ} \mathrm{C}$ for $30 \mathrm{~s}$. The relative elvels of target gene mRNA transcripts to control GAPDH were calculated by $2-\triangle \Lambda C T$.

\section{Statistical Analysis}

All data are expressed as means \pm S.D. The difference among groups was tested by one-way ANOVA followed by Scheffe's modified F-test for multiple comparisons using SPSS 17.0. A $P$ value of $<0.05$ was considered statistically significant.

\section{Results}

Treatment with Minocycline Reduces Urine amount and Urinary Proteins in Rats with DN

To test the effect of minocycline treatment, groups of DN and control rats were treated with minocycline daily for 16 weeks and their urine volumes were detected longitudinally (Table 1). We observed that treatment with minocycline in non-diabetic rats did not significantly change the amounts of urine through the 16-week observation period, treatment with minocycline for 4 weeks significantly reduced the amounts of urine in the DN rats, as compared with that in the DN group of untreated rats. Continual treatment with minocycline further gradually reduced the urinary volumes in the DN rats. Interestingly, treatment with minocycline beginning at 8 weeks post the onset hyperglycemia also significantly reduced the volumes of urine in the DN rats. Similar patterns of the excretion of urinary proteins were detected in the different groups of rats through the observation period (Table 2). Similarly, treatment with minocycline in healthy rats did not modulate significantly the levels of serum creatinine (Scr) and BUN while treatment with minocycline significantly reduced the levels of Scr and BUN in DN rats, even beginning treatment at 8 weeks post the onset hyperglycemia (Table 3). However, minocycline did not alter the concentrations of ALT among these groups of rats. These data clearly demonstrated that treatment with minocycline therapeutically improved polyurine, proteinuria and mitigated DN-related kidney impairment in DN rats.

Table 1. Effect of minocycline on $24 \mathrm{~h}$ urine volume in DN rats.

\begin{tabular}{|c|c|c|c|c|c|}
\hline Group & $\mathrm{n}$ & Week 4 & Week 8 & Week 12 & Week 16 \\
\hline $\mathrm{N}$ & 10 & $12.75 \pm 1.70$ & $11.00 \pm 4.96$ & $15.00 \pm 2.58$ & $12.00 \pm 5.718$ \\
\hline D & 10 & $233.80 \pm 24.20^{\Delta}$ & $242.20 \pm 20.53^{\Delta}$ & $297.00 \pm 16.76^{\Delta}$ & $297.40 \pm 44.98^{\Delta}$ \\
\hline NM & 10 & $15.25 \pm 1.25$ & $12.50 \pm 2.08$ & $17.00 \pm 4.32$ & $10.75 \pm 4.57$ \\
\hline M4 & 10 & $170.00 \pm 16.95^{* \Delta}$ & $122.55 \pm 28.54^{* \triangle}$ & $119.33 \pm 25.86^{\circ}$ & $108.33 \pm 6.36^{* \Delta}$ \\
\hline M2 & 10 & $286.80 \pm 63.51^{* \triangle}$ & $288.60 \pm 41.09^{* \Delta}$ & $156.00 \pm 4.94^{* \Delta}$ & $114.80 \pm 9.12^{* \Delta}$ \\
\hline
\end{tabular}

Data are the means $\pm \mathrm{SD}$ of $\mathrm{ml} / 24 \mathrm{~h}$ of each group $(\mathrm{n}=10)$; *, $\mathrm{P}<0.05$ vs. the $\mathrm{D}$ group; $\Delta$, $\mathrm{P}<0.05$ vs. the $\mathrm{N}$ group. 
Table 2. Effect of minocycline on urine protein after STZ injection.

\begin{tabular}{lrrrrr}
\hline Group & $\mathrm{n}$ & Week 4 & Week 8 & Week 12 & \multicolumn{1}{c}{ Week 16 } \\
\hline $\mathrm{N}$ & 10 & $0.0066 \pm 0.0028^{*}$ & $0.0050 \pm 0.0030^{*}$ & $0.0057 \pm 0.0025^{*}$ & $0.0051 \pm 0.0016^{*}$ \\
$\mathrm{D}$ & 10 & $0.2053 \pm 0.1702^{\Delta}$ & $0.2505 \pm 0.0460^{\Delta}$ & $0.2860 \pm 0.1259^{\Delta}$ & $0.3015 \pm 0.1066^{\Delta}$ \\
$\mathrm{NM}$ & 10 & $0.0059 \pm 0.0004^{*}$ & $0.0045 \pm 0.0038^{*}$ & $0.0052 \pm 0.0009^{*}$ & $0.0059 \pm 0.0025^{*}$ \\
$\mathrm{M} 4$ & 10 & $0.0957 \pm 0.0183^{*}$ & $0.0820 \pm 0.0189^{*}$ & $0.0782 \pm 0.0303^{*}$ & $0.0877 \pm 0.0163^{*}$ \\
$\mathrm{M} 2$ & 10 & $0.2225 \pm 0.0601^{\Delta}$ & $0.2915 \pm 0.0421^{\Delta}$ & $0.0853 \pm 0.0432^{*}$ & $0.0828 \pm 0.0275^{*}$ \\
\hline
\end{tabular}

mean \pm s.d., $g / 24 h \cdot L ; *, P<0.05$, as compared to the $\mathrm{D}$ group; $\Delta, \mathrm{P}<0.01$, as compared to the $\mathrm{N}$ group $\mathrm{P}<0.05$.

Table 3. Effect of minocycline on Scr, BUN and ALT 16 weeks after STZ injection

\begin{tabular}{ccccc}
\hline Group & $\mathrm{n}$ & $\mathrm{Scr}(\mu \mathrm{mon} / \mathrm{L})$ & $\mathrm{BUN}(\mathrm{mmol} / \mathrm{L})$ & ALT $(\mathrm{U} / \mathrm{L})$ \\
\hline $\mathrm{N}$ & 9 & $41.50 \pm 1.73$ & $7.12 \pm 0.80$ & $39.25 \pm 7.50$ \\
$\mathrm{D}$ & 10 & $150.00 \pm 23.50^{\triangle}$ & $17.82 \pm 1.13^{\triangle}$ & $37.00 \pm 5.24$ \\
$\mathrm{NM}$ & 9 & $47.00 \pm 1.41$ & $6.97 \pm 0.43$ & $39.25 \pm 7.50$ \\
M4 & 9 & $78.88 \pm 5.68^{\star \Delta}$ & $11.48 \pm 2.96^{*}$ & $43.33 \pm 10.89$ \\
M2 & 9 & $76.60 \pm 5.41^{\star \Delta}$ & $12.82 \pm 2.11^{*} \triangle$ & $43.00 \pm 4.79$
\end{tabular}

Data are the means \pm SD of each group; ${ }^{*}, \mathrm{P}<0.05$ vs. the $\mathrm{D}$ group; $\Delta, \mathrm{P}<0.05$ vs. the $\mathrm{N}$ group.

\section{Effect of Minocycline Intervention on Renal Histopathology}

To understand the action of minocycline, we characterized kidney tissues and found that while there was no obvious abnormality in the kidney histology in the $\mathrm{N}$ and $\mathrm{NM}$ groups of rats, there were clear abnormalities in the glomeruli of the DN rats (Fig. 1). There were obvious collapse of the glomerular capillaries, increased glomerular volume and thickness of basement membrane, slightly expanded mesangial areas with plumped PAS stained area, and vacuolar degeneration/hyalinization of renal tubular epithelial cells in the D group of rats. However, following treatment with minocycline, there were less DN-related pathogenic changes in the kidney tissues. Quantitative analysis of the glomerular areas indicated that there was no significant difference in the glomerular areas among these groups of rats (Table 4). These data indicated that treatment with minocycline mitigated DN-related kidney injury in rats.

Table 4. Effect of minocycline on area of glomerular in DN rats

\begin{tabular}{ccc}
\hline Group & $\mathrm{n}$ & Area of glomenular $\left(\operatorname{mean} \pm\right.$ s.d., $\left.\mu \mathrm{M}^{2}\right)$ \\
\hline N & 10 & $4299.838 \pm 163.195$ \\
D & 10 & $4849.207 \pm 823.438$ \\
NM & 10 & $4419.158 \pm 288.456$ \\
M4 & 10 & $4227.661 \pm 343.737$ \\
M2 & 10 & $4535.102 \pm 147.100$ \\
\hline
\end{tabular}



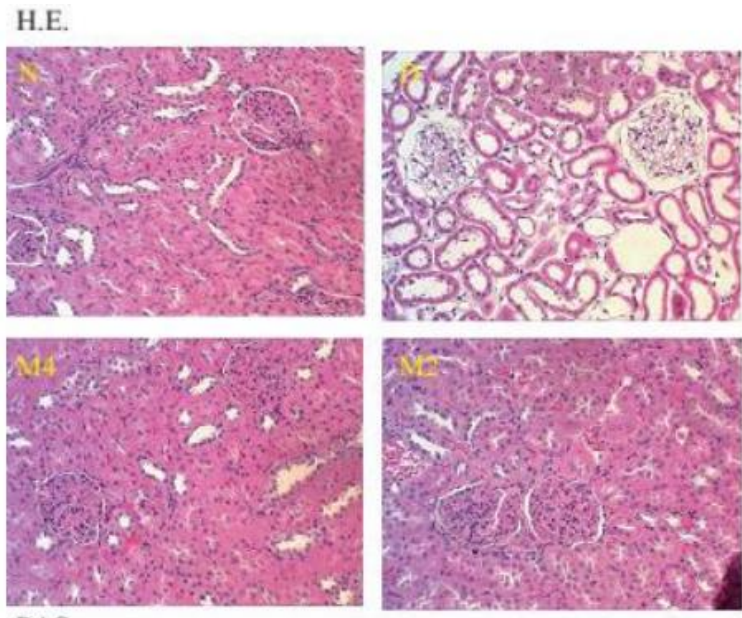

PAS
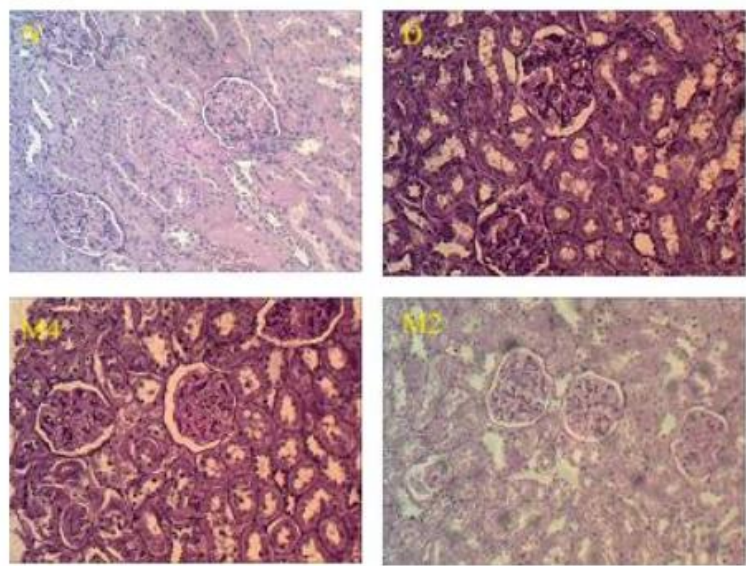

Fig. 1. Minocycline prevents glomerulosclerosis. As sessment of glomerular pathology by H.E. staining and periodic acid-Schiff(PAS) staining. Amplification: 200 times.

\section{Effect of Minocycline on Apoptosis of Kidney Cells in DN rats}

As shown in the TUNEL assay (Fig. 2, Table5), the number of kidney apoptotic cells was significantly increased in all the STZ treated rats (D, M2 and M4 group) compared with the normal control rats (N group). Nearly no TUNEL-positive cell was observed in kidney of control rats. There were more TUNEL-positive cells in the NM group than that of $\mathrm{N}$ group. There was no significant difference between NM group and $\mathrm{N}$ group $(\mathrm{p}>0.05)$. The increased apoptotic cells within glomeruli in $\mathrm{D}$ group rats were significantly abrogated by treatment with minocycline (M2 or M4 group, $\mathrm{p}<0.05)$. Treatment with minocycline for 8 weeks (M2) and 16 weeks (M4) had a similiar effect on glomerular cell apoptosis.

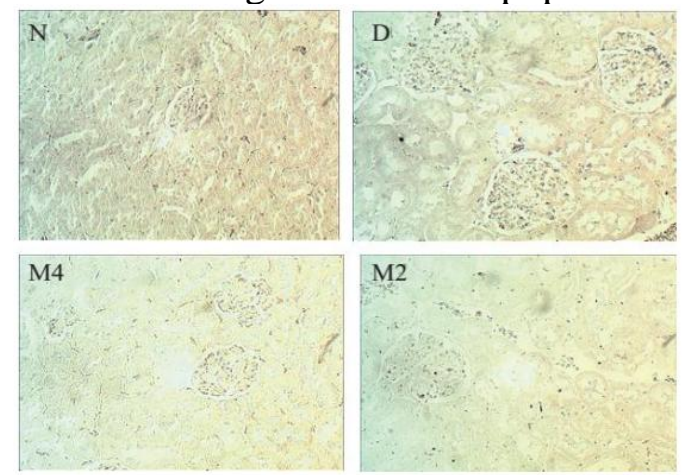

Fig. 2. Assessment of apoptotic cells in glomerular by TUNEL staining. Amplification: 200 times. 
Table 5. The rates of renal cell apoptos is in each group

\begin{tabular}{ccc}
\hline Group & $\mathrm{n}$ & Cell apoptosis rate \\
\hline $\mathrm{N}$ & 10 & $2.500 \pm 0.430^{*}$ \\
$\mathrm{D}$ & 10 & $16.133 \pm 1.145$ \\
$\mathrm{NM}$ & 10 & $3.833 \pm 0.577^{*}$ \\
$\mathrm{M} 4$ & 10 & $8.777 \pm 1.258^{*}$ \\
M2 & 10 & $10.266 \pm 1.164^{*}$ \\
\hline
\end{tabular}

*, $\mathrm{P}<0.05$, as compared to the $\mathrm{D}$ group

\section{Relative mRNA Expression Level of Caspase-3, Bcl-2 and Bax in DN Rats}

To further understand the action of minocycline, we characterized the relative levels of caspase 3 , BcL-2 and Bax mRNA transcripts to control GAPDH in the kidney tissues by quantitative RT-PCR (Table 6). We found that after the rats were sacrificed, the mRNA expression of caspase-3, Bcl-2 and Bax in kidney was detected (Table 6). The Bcl-2 mRNA expression in the $\mathrm{N}$ group was relatively low, while its expression was higher in the NM group but not significant $(\mathrm{p}>0.05)$. The Bcl-2 mRNA expression in the $\mathrm{D}$ group is significantly lower than that of $\mathrm{N}$ group ( $\mathrm{p}<0.05$ ), while there was no significant difference of Bcl-2 mRNA level between the $\mathrm{N}$ group and M2 or M4 group. The mRNA levels of Bcl-2 in the M2 and M4 are significantly higher that of $\mathrm{D}$ group $(\mathrm{p}<0.05)$. The Bax mRNA in the $\mathrm{D}$ group is significantly higher than that in the $\mathrm{N}$ group $(\mathrm{P}<0.05)$. The Bax mRNA level in the M2 or M4 group is lower than $\mathrm{D}$ group $(\mathrm{P}<0.05)$. Furthermore, the expression of caspase $3 \mathrm{mRNA}$ in the $\mathrm{D}$ group is higher that $\mathrm{N}$ or $\mathrm{NM}$ group $(\mathrm{p}<0.05)$, while there was no significant difference between M2 and M4 group. The minocycline treatment significantly reduced the caspase- 3 mRNA level in both the M2 and M4 group as compared to the D group $(\mathrm{p}<0.05)$.

Table 6. Relative mRNA expression level of caspase-3, Bcl-2 and Baxin DN rats after minocycline treatment.

\begin{tabular}{|c|c|c|c|c|}
\hline Group & $\mathrm{n}$ & caspase-3 & $\mathrm{Bcl}-2$ & Bax \\
\hline $\mathrm{N}$ & 10 & $4.995 \pm 1.375 *^{\#}$ & $0.873 \pm 0.441{ }^{\# \#}$ & $1.020 \pm 0.117^{* \#}$ \\
\hline $\mathrm{D}$ & 10 & $1.025 \pm 0.265$ & $0.938 \pm 0.080$ & $7.422 \pm 3.764$ \\
\hline NM & 10 & $9.663 \pm 4.026^{\#}$ & $0.320 \pm 0.182$ & $1.880 \pm 0.939 *$ \\
\hline M4 & 10 & $1.582 \pm 1.135 * \#$ & $1.100 \pm 0.633 *^{\#}$ & $2.974 \pm 1.149 * \#$ \\
\hline M2 & 10 & $5.059 \pm 2.192 * \#$ & $0.755 \pm 0.450 *^{\#}$ & $3.327 \pm 0.876 * \#$ \\
\hline
\end{tabular}

mean \pm s.d., *, $\mathrm{P}<0.05$, as compared to the $\mathrm{D}$ group, \#, $\mathrm{P}<0.05$, as compared to the $\mathrm{D}$ group. 


\section{Discussion}

The present study provided evidence that minocycline can decrease urine volume, proteinuria, BUN, serum creatinine, and reduced glomerular apoptosis in STZ induced DN rats. STZ have induced caspase- 3 and reduced Bcl-2 and Bax mRNA expression in the kidney of the DN rats, while minocycline can reverse the abnormal expression of these genes indicate that the renoprotective action of minocycline is probably attributable to an anti-apoptosis effect.

Urine protein level, BUN, and Scr are known as indicators for renal dysfunction[23]. First, we successfully develop the symptoms of DN in our STZ induced rat model. In fact, proteinuria indicates a decline of renal function[24,25] and it is known that STZ injection causes proteinuria, as shown previously[25,26]. In our study, urinary protein excretions after STZ treatments were significantly higher than that of control and minocycline only treated rats. The progress of pathology in STZ-treated groups was more advanced than that of normal groups. Then, the effect of minocycline on STZ-injured renal function was investigated. Minocycline alleviated the progress of nephrotic syndrome, as evidenced by Scr and urinary protein levels compared with the values at baseline, while minocycline did not affect these parameters in the non-STZ treated rats. Pathological analysis also showed that the necrosis and number of apoptotic cell in the kidney were reduced in the minocycline treated STZ-induced DN rats. Furthermore, the results showed non-significant differences in the serum levels of ALT in all studied groups in comparison to normal control group, which indicated that minocycline did not induce increase in hepatic enzymes. Therefore, it is possible to suggest that minocycline is safe in DN rats and protect the function of the DN rats by inhibiting the apoptosis of kidney cells in the DN rats.

It is well known that injury to podocyte foot processes in the kidney causes protein excretion into the urine and the injury and apoptosis of podocytes play a crucial role in the progress of DN[27]. The caspase family of cysteine proteases plays a key role in apoptosis[28]. With inductions of endogenous or exogenous signals, the activated caspase mediates apoptosis by proteolysis of specific substrates[29]. Caspase activation functions as the evoking caspase-like effect, which results in triggering of cellular apoptosis[30]. There are two kinds of caspases involved in apoptosis, one is initiators which is activated by cellular receptor, and the other is effectors activated by mitochondrial permeability transition. Pro-apoptotic signals activate initiator caspases such as caspase- 8 , which then activate the process effector caspases such as caspase-3, leading to cell death[31]. The expression of caspase- 3 was up-regulated in the STZ treated DN rats, while minocycline attenuated the up-regulated caspase- 3 expression in the DN rats which suggesting that minocycline may target the caspase- 3 in DN rats.

Bcl-2 family proteins are important regulators of apoptosis[32,33]. To identify the possible molecular changes that are responsible for the renal protective effect of minocycline, we investigated the $\mathrm{Bcl}-2$ family proteins. Bcl-2 directly interacts with the proapoptotic molecules in the mitochondrion to antagonize apoptosis[34,35]. The interaction between the Bcl-2 protein and the proapoptotic proteins may determine the fate of a cell. Thus, we first detected the expression of Bcl-2 in the kidney of STZ and non-STZ treated rats with or without minocycline-treatment. Significantly higher Bcl-2 mRNA was expressed in minocycline-treated kidney. There are three extensively characterized mammalian Bcl-2 family members are the antiapoptotic genes Bcl-2 and Bcl-XL, and the proapoptotic gene bax. Individual family members can function to either block or promote programmed cell death[36]. Among them, Bax is the only one to promote cell death. To determine the bax expression in 
minocycline-treated cells, the expression of bax in the kidney was detected by quantitiative PCR. The expression of bax mRNA was induced in the STZ treated kidney and reduced by the minocycline treatment. Interestingly, previous reports showed that minocycline prevented the upregulation of p53 and Bax following ischemia 13). Thus minocycline protected the STZ injured kidney by inhibiting both the caspase- 3 and Bax but stimulating the expression of Bcl-2 the expression which normally execute the apoptotic program.

\section{Summary}

In conclusion, the present study indicated that minocycline exhibited kidney protective effects in DN rats. These effects are attributed, at least partly, to the anti-apoptotic activity in the kidney while improving blood and urine parameters and protecting kidney tissues. However, the precise molecular mechanism of these effects remains to be elucidated.

\section{References}

[1] Guay C, Roggli E, Nesca V, Jacovetti C, Regazzi R: Diabetes mellitus, a microRNA-related disease? Transl Res, 157, 253-264 (2011).

[2] Ritz E, Schmieder RE, Pollock CA: Renal protection in diabetes: lessons from ONTARGET. Cardiovasc Diabetol, 9, 60 (2010).

[3] WolfG, Chen S, Ziyadeh FN: From the periphery of the glomerular capillary wall toward the center of disease: podocyte injury comes of age in diabetic nephropathy. Diabetes, 54, 1626-1634 (2005).

[4] Stitt-Cavanagh E, MacLeod L, Kennedy C: The podocyte in diabetic kidney disease. ScientificWorldJournal, 9, 1127-1139 (2009).

[5] Mundel P, Reiser J: Proteinuria: an enzymatic disease of the podocyte? Kidney Int, 77, 571-580 (2010).

[6] Jefferson JA, Shankland SJ, Pichler RH: Proteinuria in diabetic kidney disease: a mechanistic viewpoint. Kidney Int, 74, 22-36 (2008).

[7] Drummond K, Mauer M: The early natural history of nephropathy in type 1 diabetes: II. Early renal structural changes in type 1 diabetes. Diabetes, 51, 1580-1587 (2002).

[8] Menini S, Iacobini C, Oddi G, RicciC, Simonelli P, Fallucca S, Grattarola M, Pugliese F, Pesce C, Pugliese G: Increased glomerular cell (podocyte) apoptosis in rats with streptozotocin-induced diabetes mellitus: role in the development of diabetic glomerular disease. Diabetologia, 50, 2591-2599 (2007).

[9] Sohn E, Kim J, Kim CS, Kim YS, Jang DS, Kim JS: Extract of the aerial parts of Aster koraiensis reduced development of diabetic nephropathy via anti-apoptosis of podocytes in streptozotocin-induced diabetic rats. Biochem Biophys Res Commun, 391, 733-738 (2010).

[10] Klein NC, Cunha BA: Tetracyclines. Med Clin North Am, 79, 789-801 (1995).

[11]Ryan ME, Greenwald RA, Golub LM: Potential of tetracyclines to modify cartilage breakdown in osteoarthritis. Curr Opin Rheumatol, 8, 238-247 (1996).

[12] Arvin KL, Han BH, Du Y, Lin SZ, Paul SM, Holtzman DM: Minocycline markedly protects the neonatal brain against hypoxic-ischemic injury. Ann Neurol, 52, 54-61 (2002). 
[13] Chen M, Ona VO, Li M, Ferrante RJ, Fink KB, Zhu S, Bian J, Guo L, Farrell LA, Hersch SM, Hobbs W, Vonsattel JP, Cha JH, Friedlander RM: Minocycline inhibits caspase- 1 and caspase- 3 expression and delays mortality in a transgenic mouse model of Huntington disease. Nat Med, 6, 797-801 (2000).

[14] Du Y, Ma Z, Lin S, Dodel RC, Gao F, Bales KR, Triarhou LC, Chernet E, Perry KW, Nelson DL, Luecke S, Phebus LA, Bymaster FP, Paul SM: Minocycline prevents nigrostriatal dopaminergic neurodegeneration in the MPTP model of Parkinson's disease. Proc Natl Acad Sci U S A, 98, 14669-14674 (2001).

[15] Sanchez Mejia RO, Ona VO, Li M, Friedlander RM: Minocycline reduces traumatic brain injury-mediated caspase- 1 activation, tissue damage, and neurological dysfunction. Neurosurgery, 48, 1393-1399; discussion 1399-1401 (2001).

[16]Zhu S, Stavrovskaya IG, Drozda M, Kim BY, Ona V, Li M, Sarang S, Liu AS, Hartley DM, Wu DC, Gullans S, Ferrante RJ, Przedborski S, Kristal BS, Friedlander RM: Minocycline inhibits cytochrome $\mathrm{c}$ release and delays progression of amyotrophic lateral sclerosis in mice. Nature, 417, 74-78 (2002).

[17]Popovic N, Schubart A, Goetz BD, Zhang SC, Linington C, Duncan ID: Inhibition of autoimmune encephalomyelitis by a tetracycline. Ann Neurol, 51, 215-223 (2002).

[18] Wang X, Zhu S, Drozda M, Zhang W, Stavrovskaya IG, Cattaneo E, Ferrante RJ, Kristal BS, Friedlander RM: Minocycline inhibits caspase-independent and -dependent mitochondrial cell death pathways in models of Huntington's disease. Proc Natl Acad Sci U S A, 100, 10483-10487 (2003).

[19]Lin S, Wei X, Xu Y, Yan C, Dodel R, Zhang Y, Liu J, Klaunig JE, Farlow M, Du Y: Minocycline blocks 6-hydroxydopamine-induced neurotoxicity and free radical production in rat cerebellar granule neurons. Life Sci, 72, 1635-1641 (2003).

[20] Wang J, Wei Q, Wang CY, Hill WD, Hess DC, Dong Z: Minocycline up-regulates Bcl-2 and protects against cell death in mitochondria. J Biol Chem, 279, 19948-19954 (2004).

[21]Cheung PY, Sawicki G, Wozniak M, Wang W, Radomski MW, Schulz R: Matrix metalloproteinase-2 contributes to ischemia-reperfusion injury in the heart. Circulation, 101, 1833-1839 (2000).

[22] Nieman GF, Zerler BR: A role for the anti-inflammatory properties of tetracyclines in the prevention of acute lung injury. Curr Med Chem, 8, 317-325 (2001).

[23]Camici M: The Nephrotic Syndrome is an immunoinflammatory disorder. Med Hypotheses, 68, 900-905 (2007).

[24] Javaid B, Olson JL, Meyer TW: Glomerular injury and tubular loss in adriamycin nephrosis. J Am Soc Nephrol, 12, 1391-1400 (2001).

[25] He L, Rong X, Jiang JM, Liu PQ, Li Y: Amelioration of anti-cancer agent adriamycin-induced nephrotic syndrome in rats by Wulingsan (Gorei-San), a blended traditional Chinese herbal medicine. Food Chem Toxicol, 46, 1452-1460 (2008).

[26]Chen ST, Peng SJ, Chen JR: Effects of dietary protein on renal function and lipid metabolism in five-sixths nephrectomized rats. Br J Nutr, 89, 491-497 (2003). 
[27] Kramer AB, Bos $H$, van Goor H, Navis GJ: Sodium intake modifies the negative prognostic value of renal damage prior to treatment with ACE inhibitors on proteinuria induced by adriamycin. Nephron Physiol, 103, p43-52 (2006).

[28] Wilson MR: Apoptosis: unmasking the executioner. Cell Death Differ, 5, 646-652 (1998).

[29] Mishra R, Emancipator SN, Kern T, Simonson MS: High glucose evokes an intrinsic proapoptotic signaling pathway in mesangial cells. Kidney Int, 67, 82-93 (2005).

[30] Wang J, Lenardo MJ: Roles of caspases in apoptosis, development, and cytokine maturation revealed by homozygous gene deficiencies. J Cell Sci, 113 (Pt 5), 753-757 (2000).

[31] Thorburn A: Death receptor-induced cell killing. Cell Signal, 16, 139-144 (2004).

[32 Estaquier J, Vallette F, Vayssiere JL, Mignotte B: The mitochondrial pathways of apoptosis. Adv Exp Med Biol, 942, 157-183 (2012).

[33] Adams JM, Cory S: The Bcl-2 protein family: arbiters of cell survival. Science, 281, 1322-1326 (1998).

[34] Mahajan NP, Linder K, Berry G, Gordon GW, Heim R, Herman B: Bcl-2 and Bax interactions in mitochondria probed with green fluorescent protein and fluorescence resonance energy transfer. Nat Biotechnol, 16, 547-552 (1998).

[35]Zhang L, Xing D, Chen M: Bim(L) displacing Bcl-x(L) promotes Bax translocation during TNFalpha-induced apoptosis. Apoptosis, 13, 950-958 (2008).

[36] Dong Z, Wang J: Hypoxia selection of death-resistant cells. A role for Bcl-X(L). J Biol Chem, 279, 9215-9221 (2004). 